\title{
La Educación Comparada como método para la mejora y la visualización de la Educación Musical
}

\author{
Mateu-Luján, Borja \\ Universitat de València, València, España \\ Borja.Mateu@uv.es
}

Alventosa-Bleda, Ester

Universitat de València, València, España

maria.e.alventosa@uv.es

\section{Resumen}

El detrimento de la presencia de la Educación Musical en los diferentes niveles educativos en España es un hecho contrastado. Si nos fijamos en el transcurso de las numerosas leyes educativas que se han desarrollado en nuestro país podemos observar como poco a poco la música ha ido perdiendo relevancia. La principal causa de este hecho se deriva, entre otras, de la capitalización de la educación. Para combatir este efecto una de las mejores maneras es analizar cómo se van redefiniendo en otros países y regiones los diferentes sistemas educativos haciendo frente a estas adversidades. Un modo de crear estos espacios en los que compartir conocimiento y crear puntos de unión en relación con un mismo problema, sin duda es a través de la proliferación de estudios de Educación Comparada desde la perspectiva Musical.

En este texto se presenta cuál es el estado de la cuestión y qué perspectivas de futuro deben desarrollarse para la mejora y mayor presencia de la Educación Musical en los sistemas educativos. En referencia a la temática del congreso este texto está totalmente ligado puesto que existe gran variedad de experiencias en las que la música a través de su poder cohesionador y potenciador de las diferentes capacidades humanas ayuda a fomentar la Inclusión y la Equidad.

\section{Abstract}

The detriment of the presence of Music Education in the different educational levels in Spain is a contrasted fact. If we look at the course of the numerous educational laws that have been developed in our country, we can see how little by little music has been losing relevance. The main cause of this fact is derived, among others, from the capitalization of education. To combat this effect, one of the best ways is to analyze how different educational systems are redefining in other countries and regions facing these adversities. A way to create these spaces in which to share knowledge and create points of union in relation to the same problem, without a doubt, is through the proliferation of studies of Comparative Education from the Musical perspective.

This text presents the state of the question and what future perspectives should be developed for the improvement and greater presence of Music Education in educational systems. In reference to the topic of the congress this text is totally linked because there are a great variety of experiences in which music through its cohesive power and enhancer of different human capacities helps to promote Inclusion and Equity.

Palabras clave: Educación Comparada, Educación Musical, Sistemas Educativos, Metodología, Globalización.

Keywords: Comparative Education, Music Education, Educational Systems, Methodology, Globalization. 


\section{INTRODUCCIÓN}

La metodología comparada es utilizada en diversos campos científicos, aunque en muchos casos no se tenga conciencia de ello. «En los últimos 25 años, el Análisis Cualitativo Comparativo ha estado en el centro de atención de la metodología de las Ciencias Sociales. Se fundamenta en el conjunto de relaciones y objetivos establecidos en el descubrimiento de condiciones suficientes y necesarias -y sus derivadas» (Wagemann, 2014). Las Ciencias de la Educación, como Ciencias Sociales, no han sido ajenas a este hecho, y la Educación Comparada se ha convertido en una metodología la cual ha alcanzado un gran estatus. Está «presente en las revistas de mayor impacto científico del país, cercana a los grandes núcleos universitarios y con un impacto cada vez mayor, estamos ante una disciplina que sigue en auge y que se consolida en el panorama nacional español» (Álvarez López, 2015).

Una de las causas de este incremento de la Educación Comparada ha sido debido a que los gobiernos reconocen que el éxito futuro de sus países, incluso su supervivencia, en el mercado global depende menos de sus recursos naturales que de sus recursos humanos (Watson, 2001). En consecuencia, la educación toma especial relevancia como la principal manera para aumentar los recursos humanos de cada país. «En una era donde las preocupaciones sobre la globalización atraviesan los debates académicos y populares, parece particularmente apremiante que los cursos de educación comparada e internacional dediquen cierta atención a lidiar con las formas en que las escuelas están situadas en, y en relación con, procesos y fenómenos globales» (Manzon, 2016). De este modo, la Educación Comparada se ha convertido en una metodología que permite tanto solventar problemas relacionados tanto con la inclusión y la equidad, como estudiar aspectos concretos en diferentes países o regiones con el fin de adoptar medidas que ayuden a solucionar determinados problemas en otras zonas geográficas o aumentar la calidad de la educación.

La utilidad de la Educación Comparada y el número de publicaciones que ayudan a sistematizar el método, pueden ser utilizados por otros campos de estudio a los que, debido a la proximidad a la Educación, podrían beneficiarse de esta metodología. Además, estos campos de estudio de la misma forma ayudarían a reflexionar, mejorar y dar difusión a esta metodología. En concreto hablamos de la enseñanza de las diferentes materias que componen el currículum de los diferentes planes de estudio de las diferentes etapas educativas de cada país. Son materias que por sí solas no tienen ninguna relación, pero cuando se investiga su docencia, la Educación Comparada puede ser muy interesante para conocer como se resuelven problemas similares relacionados con la educación en otros contextos ya sea en perspectiva intranacional, supranacional o internacional.

Esto no quiere decir que no se realicen trabajos comparativos en este ámbito de estudio. Al contrario, existen numerosos trabajos de gran calidad que comparan técnicas, metodologías, currículums, formación del profesorado, planes de estudio... Sin embargo, sería adecuado que estos trabajos se realizaran desde una perspectiva de la Educación Comparada con el fin de aprovechar las numerosas reflexiones ya producidas sobre aspectos derivados de las comparaciones en educación. Entre estos campos de estudio encontramos la Educación Musical la cual necesita sin duda, de la Educación Comparada para competir, entre otras contrariedades, contra el detrimento de la presencia de la materia de música en la Educación Obligatoria y para mejorar la formación de los docentes que impartan estas materias. En el caso de España estas dos contrariedades son muy preocupantes. Por un lado,

Con la aprobación en 2013 (9 de diciembre) de la Ley Orgánica para la mejora de la Calidad Educativa (LOMCE), se elimina la obligatoriedad de cursar enseñanzas artísticas en Educación Primaria, dejando en manos de las administraciones autonómicas la total competencia en estas materias, la educación artística (primaria) y la música (secundaria) pasan a considerarse <asignaturas optativas>, podrán ser cursadas en función de la regulación y de la programación de la oferta educativa que establezca cada administración educativa y, en su caso, de la oferta de los centros docentes. (Morales, 2017, p.391).

Por otro lado, con la introducción de los nuevos planes de estudio de magisterio:

Si bien se conseguía una antigua reivindicación de las Escuelas de Magisterio de convertir los títulos de maestro en títulos equivalentes a las antiguas licenciaturas, un nuevo cambio de paradigma obliga a la formación de maestros generalistas con una mínima especialización frente a la formación de maestros especialistas que se había producido con la aplicación de la LOGSE en 1991. (Morales, 2017). 
Aspectos que la Educación Comparada puede aportar visiones holísticas de como se afrontan estas tendencias internacionales de disminución de la presencia y la calidad de la Educación Musical en otros países. Sobre todo, en países en los que la Educación Musical tiene tradicionalmente una gran presencia en el Sistema Educativo.

\section{LA EDUCACIÓN COMPARADA APLICADA A LA EDUCACIÓN MUSICAL}

La conexión entre Educación Comparada y Educación Musical no es un aspecto novedoso ya que existen algunas publicaciones que relacionan estos dos campos de estudio. Desde esta perspectiva es conveniente destacar los estudios de Arnold Bentley como uno de los pioneros de la Educación Comparada aplicada a la Educación Musical. En 1968 el Dr. Arnold Bentley, en la Universidad de Reading, Inglaterra realizaba investigaciones sobre metodología para la investigación en educación musical. Esta «abarcó elementos de educación comparada y de metodología para la investigación experimental de la percepción del fenómeno sonoro» (Frega, 1997). A partir de estas investigaciones fueron diversas las que se han ido uniendo a estas pesquisas que engloban estos dos campos de estudio.

«Para el análisis y la comparación de la educación musical en los sistemas nacionales, se puede usar la estructura conceptual adaptada del trabajo de Holmes (1981) y Bereday (1966 y 1967)» (Lepherd, 1993). Los trabajos de Holmes y Bereday son trabajos, bien conocidos por los comparatistas de educación, por lo que se muestra la gran utilidad de la Educación Comparada, para algunos investigadores en Educación Musical. Aunque, si bien cada vez aparecen más estudios de este tipo, es destacable la evolución de la nomenclatura de estudio. Estos estudios cuando exponen su metodología, en muchas ocasiones, ya no hablan de Educación Comparada, sino que se utiliza la metodología «Educación Comparada Musical» (Comparative Music Education).

«Aunque la Educación Comparada Musical no es completamente aceptada como un campo explícito de investigación» (Kertz-Welzel, 2008); lo cierto es que, la estandarización de este nuevo término crea como mínimo una matización que hace distanciar la Educación Comparada de la Educación Comparada Musical. Es lógico que los estudios comparados se deben realizar por investigadores con un gran conocimiento de los objetos que se confrontan. La educación es un ámbito de estudio muy amplio y no todos los investigadores tienen conocimientos sobre todo los elementos. No obstante, se hace complicado fragmentar dos campos de estudio tan próximos. Así, del mismo modo que la Educación Comparada, la Educación Comparada Musical «plantea algunas preguntas acerca de cómo, aquellas ideas encontradas en una situación dada, pueden ser adoptadas y aplicadas exitosamente en otra situación, y cómo se pueden hacer las comparaciones para que las conclusiones alcanzadas sean confiables y válidas (Lepherd, 1993).

\section{PROBLEMAS DE LA EDUCACIÓN MUSICAL QUE SE PUEDEN ABORDAR DESDE LA EDUCACIÓN COMPARADA}

Tal y como hemos podido observar en la introducción la Educación Musical se enfrenta a grandes retos, ya que su presencia y su calidad están disminuyendo en el sistema educativo español. Junto a estos retos, existen muchos otros que podrían resolverse desde una perspectiva comparada. A continuación, se exponen algunas líneas de investigación en las cuáles sería útil enfocar los trabajos científicos para mejorar la Educación Musical.

- Educación musical en la etapa obligatoria. La inteligencia musical es una fuente muy importante de desarrollo emocional, social y comunicativo, para todo tipo de estudiante (García-Vélez y Maldonado Rico, 2017). Como se puede observar, el desarrollo de la música ayuda a desarrollar algunas de las competencias básicas. Sin embargo, sería oportuno realizar un estudio comparado de como los países con más presencia musical, integran la educación musical en sus diferentes sistemas educativos con el fin de desarrollar estas habilidades en los alumnos.

- Formación de docentes de música en otros países. Posiblemente, esta vía de investigación sea la que más desarrollada se encuentra en estos momentos. La discusión sobre si se deben formar docentes especialistas en una o varias materias o por el contrario los docentes deben ser polivalentes, está presente actualmente. Este debate implica tanto a músicos como a investigadores de la educación en general. 
- El currículum de la Educación Musical Obligatoria. La Educación Musical no suele tener la categoría de área curricular en los sistemas educativos, sino que se configura como una materia. En el sistema educativo español en concreto la Educación Musical como la Educación Plástica configuran el área artística. Esto provoca por ejemplo que los estudios comparados realizados por las organizaciones europeas e internacionales no analicen la Educación Musical, sino que analizan el área artística. Es por ello, que son necesarios estudios comparados que analicen únicamente la Educación Musical.

- La Educación Musical Superior. Sin duda la Educación Musical Superior es uno de los grandes retos que pueden y deben ser afrontados desde la Educación Comparada. En la mayoría de los países europeos a excepción de Alemania la música no está presente en las Universidades, sino que se estudia en Conservatorios. Este hecho provoca que no exista investigación en este ámbito de estudio ya que están autorizados para ofrecer estudios de doctorado. Sin embargo, en países como en Estados Unidos, la música sí que pertenece al sistema universitario en todos los sentidos. Así, es interesante realizar estudios comparados entre diferentes países que sí que incluyen la música en el sistema universitario. De este modo, se puede observar como se han ido resolviendo todas y cada una de las características propias de la Educación Musical que pueden entrar en conflicto con las de la Educación Universitaria.

- Nuevos contenidos musicales. Los contenidos musicales están continuamente en evolución, es por ello por lo que, algunos países han incluido en la educación musical nuevos estilos de música, nuevos instrumentos, nuevas formas de composición. Estos contenidos se encuentran con dificultades y reticencias de las culturas en los que la música culta es predominante. La música desde esta perspectiva introduce elementos de equidad e inclusión que pueden ser amplificados con el aumento de estudios comparados que visualicen la enseñanza de diversos estilos de música representativos de diferentes culturas.

- Educación comparada aplicada a las metodologías de enseñanza de la música. Actualmente, ya existen algunos trabajos científicos que analizan y comparan los diferentes métodos de enseñanza de la música para conseguir un mejor y óptimo aprendizaje. Estos estudios se realizan sobre todo con metodologías estandarizadas. Sería conveniente sumar a estos estudios, investigaciones sobre nuevas metodologías que sean innovadoras en cada uno de los contextos.

\section{DISCUSIÓN Y CONCLUSIONES}

El futuro de la Educación Comparada y la Educación Comparada Musical es apasionante y esta lleno de buenas y prolíficas investigaciones. Después de visualizar cuál ha sido la evolución de ambos campos de estudio, sería recomendable que no se alejaran. La Educación Musical Comparada sin duda podría beneficiarse del largo camino recorrido de la Educación Comparada como ciencia. Además, la Educación Comparada dispone de un método más estandarizado el cuál puede aportar claridad a las diferentes investigaciones. Para evitar la confusión que se puede derivar entre ambos nombres, sería útil que los investigadores utilizaran como método la Educación Comparada, aunque sus textos tengan como objeto de estudio la Educación Musical. La fragmentación puede impedir la reciprocidad entre estudios que vayan en la misma línea y también la combinación interdisciplinar entre diferentes campos. Por ejemplo, si se realiza un Estudio Comparado sobre la Formación de los Maestros de matemáticas en Europa, ¿la metodología utilizada debería ser Educación Comparada Matemática? Si cada una de las áreas de conocimiento diseñara su metodología serían necesarias múltiples metodología de Educación Comparada. En consecuencia, desde nuestro punto de vista, creemos que se debe fortalecer el concepto de Educación Comparada y englobar todos los aspectos relacionados dentro de este.

Siguiendo con el ejemplo de las matemáticas, posiblemente Educación Comparada Matemática hoy en día carece de sentido debido a la buena salud que goza esta materia en todos los Sistemas Educativos. Si bien, este concepto de Educación Comparada Musical es interesante debido a necesidad 
de hacer visibles problemáticas que cada vez están más agravadas. Sin embargo, para lograr este acercamiento entre ambos estudios comparados es necesario que se fomenten las acciones colaborativas desde ambas posiciones.

\section{REFERENCIAS BIBLIOGRÁFICAS}

Álvarez López, G. (2015). La educación comparada más allá de la REEC: análisis bibliométrico de la disciplina en las cinco revistas españolas afines de más impacto entre 1995 y 2014. Revista Española de Educación Comparada, 25, 19-45.

Frega, A. L., Urtubey, P. S., \& Faculdad de Humanidades, Y. (1997). Metodología comparada de la Educación Musical.

García-Vélez, T. y Maldonado Rico, A. (2017). Reflexiones sobre la inteligencia musical. Revista española de pedagogía, 75(268), 451-461.

Kertz-Welzel, A. (2008). Music education in the twenty-first century: a cross-cultural comparison of German and American music education towards a new concept of international dialogue. Music Education Research, 10(4), 439-449.

Lepherd, L. (1993). Investigación Comparativa. En Kemp, A (Ed.) Aproximaciones a la Investigación en Educación Musical (pp. 19-30). Collegium Musicum. (Traducción A. Frega y D.P. de Graetzer).

Manzon, M. (2016). Comparative Educations to What Ends? Teaching Comparative Education: Trends and Issues Informing Practice. 
Morales, A. (2017). Presentación. La educación musical, una mirada del pasado al futuro. Revista española de pedagogía, 75 (268), 389-398.

Wagemann, C. (2014). ¿Qué hay de nuevo en el método comparado?: QCA y el análisis de los conjuntos difusos. Revista Mexicana de Análisis Político y Administración Pública, 1 (1), 51-75.

Watson, K. (2001). Introduction: Rethinking the role of comparative education. Doing comparative education research: Issues and problems, 9-20. 